\title{
Regulatory and Health Technology Assessment Considerations for Disease-Modifying Drugs in Alzheimer's Disease
}

\author{
Jacoline C. Bouvy ${ }^{1}$ (D . Pall Jonsson ${ }^{1}$. Diana O'Rourke ${ }^{1}$. Antonella Santuccione Chadha ${ }^{2} \cdot$ Niklas Hedberg $^{3}$. \\ Amr Makady ${ }^{4}$ Entela Xoxi ${ }^{5} \cdot$ Christine Gispen-de Wied ${ }^{6} \cdot$ Anja Schiel $^{7} \cdot$ Raj Long $^{8} \cdot$ John Gallacher $^{9}$
}

Published online: 22 November 2018

(c) The Author(s) 2018

\begin{abstract}
Although there are a growing number of well-reported, late-stage clinical trial failures in Alzheimer's disease, the introduction of a disease-modifying therapy within the next 5 years may be anticipated. These treatments are likely to target Alzheimer's disease in the earlier disease stages, unlike drugs that are currently available that treat symptoms of moderate-to-severe dementia. Therefore, there is a need to establish a consensus on regulatory and health technology assessment requirements for Alzheimer's disease, as a new drug will need to undergo regulatory and health technology assessments before it becomes available to patients. This article reports the discussions and activities of the regulatory and health technology assessment expert advisory group of the 2-year ROADMAP (real-world outcomes across the Alzheimer's disease spectrum: a multimodal data access platform) project. The expert advisory group discussions identified a lack of consensus on validated outcomes in the earliest Alzheimer's disease stages, the need for filling gaps between outcomes used across clinical trials and realworld settings, and the role that real-world evidence might have in characterising the impact of a possible disease-modifying therapy on caregivers, resource use and long-term outcomes.
\end{abstract}

Jacoline C. Bouvy

Jacoline.bouvy@nice.org.uk

1 Science, Policy and Research Programme, National Institute for Health and Care Excellence, 10 Spring Gardens, London SW1A 2BU, UK

2 Swissmedic, Swiss Agency for Therapeutic Products, Bern, Switzerland

3 The Dental and Pharmaceutical Benefits Agency (TLV), Stockholm, Sweden

4 Zorginstituut Nederland (ZIN), Diemen, The Netherlands

5 Faculty of Economics, Catholic University of the Sacred Heart and Graduate School of Health Economics and Management (ALTEMS), Rome, Italy

6 Medicines Evaluation Board, Utrecht, The Netherlands

7 Unit for Health Technology Assessment and Reimbursement, Department for Pharmacoeconomics, Norwegian Medicines Agency, Oslo, Norway

8 Gates Foundation, London, UK

9 University of Oxford, Oxford, UK

\section{Key Points}

Although a number of disease-modifying drugs for Alzheimer's disease are under development, none have made it to the market yet. These new treatments are targeting earlier stages of the disease and, therefore, the health technology assessment and regulatory experience with currently licensed drugs for Alzheimer's disease will only be partially applicable to the approval and reimbursement of newer drugs.

There is a need for validated and widely accepted outcomes that capture the early stages of Alzheimer's disease, including prodromal disease and mild cognitive impairment.

Real-world evidence is needed to better characterise the impact that new treatments will have on outcomes, caregivers and healthcare systems. 


\section{Introduction}

It has been more than a decade since the last drug therapy for the symptomatic treatment of dementia received a marketing authorisation in Europe. Since then, there has been a move towards the development of disease-modifying drugs in $\mathrm{Alz}$ heimer's disease (AD). We now know that AD is characterised by a long preclinical phase during which the disease starts developing in the brain prior to any detectable cognitive or functional changes [1]. Following the pre-clinical phase, there is a variable but relatively short prodromal period (typically between 4 and 5 years) when early cognitive and functional changes emerge prior to a diagnosis of AD. It is likely that initial disease-modifying treatments will be offered to people with prodromal $\mathrm{AD}$ who have positive $\mathrm{AD}$ markers (amyloid and/or tau) and mild cognitive impairment but have not yet developed sufficient functional impairment for a dementia diagnosis $[1,2]$.

Broadly, a new drug will need to demonstrate a positive benefit-risk profile before a marketing authorisation is granted by a regulatory agency such as the European Medicines Agency (EMA) or the Swiss Agency for Therapeutic Products, Swissmedic. Health technology assessment (HTA) bodies will subsequently perform assessments to advise healthcare payers whether a new drug provides added value and should be made available in a publicly funded healthcare system. As diseasemodifying drugs will be targeting earlier disease stages than currently licensed treatments, it is not apparent what type of evidence base might be required for these products to go through regulatory and HTA procedures, or whether there is an alignment in the expectations of regulators and HTAs regarding evidence supporting the value of disease-modifying drugs.

Although there is a growing number of well-reported, latestage clinical trial failures [3], the introduction of a diseasemodifying therapy within the next 5 years is anticipated. As individuals with AD generally will not have access to new drugs unless they are reimbursed by healthcare payers, there is an urgency to establish a consensus on these regulatory and HTA requirements if Europe's healthcare systems are to be ready to facilitate access to these important treatments. This article reports the discussions and activities of the regulatory and HTA expert advisory group (EXAG) of the ROADMAP (real-world outcomes across the Alzheimer's disease spectrum: a multimodal data access platform) project.

\section{ROADMAP Project}

The ROADMAP project provides the foundation for a European data platform for real-world evidence in AD. Funded through the Innovative Medicines Initiative, the 2-year project running from November 2016 to October 2018 was a public-private partnership that brought together academic partners, manufacturers (including those with disease-modifying drugs currently under development), data providers, a patient advocacy organisation, a regulatory agency and an HTA body [4]. The objective of ROADMAP was to provide the foundation for an integrated data environment and framework for real-world evidence in AD. The project consisted of different work streams, organised in work packages on the following topics: outcome definitions; identification, mapping and integration of real-world evidence; disease modelling and simulation; and health economics. ROADMAP outputs can be found on the project website (http://www. roadmap-alzheimer.org).

ROADMAP recognised that to ensure the project produces outputs that are not merely of high scientific quality but might also be usable in a regulatory and HTA context, it is paramount to consider European regulatory and HTA requirements. Therefore, ROADMAP established the EXAG consisting of regulatory and HTA experts from different European countries that provided the project with periodic input and feedback. The EXAG discussions were confidential and non-binding, and experts participated as individuals rather than as official representatives of their employers. During the course of the project, EXAG meetings were held every 3 months and different topics were discussed at these meetings, including priority outcomes across a range of stakeholders in $\mathrm{AD}$, validation of a disease-progression model currently under development and pharmacoeconomic modelling considerations.

The EXAG meetings followed a common format, with ROADMAP researchers presenting ongoing work from the different work streams, followed by discussion with the EXAG members who would comment on the findings presented. Some of the discussions focussed on a hypothetical disease-modifying therapy for AD, but none of the discussions at EXAG meetings were product specific. Minutes of all EXAG meetings were drafted and checked by the EXAG members before being finalised and shared with the ROADMAP consortium partners. For this article, we used the EXAG meeting minutes of five separate meetings that took place throughout the ROADMAP project.

\subsection{Data Cube}

ROADMAP had access to a large number of data sources including population-based databases, national registries, electronic health records (primary and secondary care), disease registries and randomised controlled trial (RCT) data. All of these data sources might contain valuable information about people with AD or dementia, but not all data sources will contain the same information or cover all the stages of the disease. ROADMAP visualised this problem as a data cube with data sources, disease stages and outcomes as the 
three axes (Fig. 1). Populating the cells of the cube for each data source enabled the available data to be described with gaps in the data becoming apparent, providing all stakeholders with a rapid and convenient summary of the evidence base.

Identifying gaps in the AD evidence base will inform scientific, clinical and regulatory activity as it will direct thinking on the collection and evaluation of further evidence. By identifying gaps now, rather than once the first disease-modifying drug becomes available, ongoing and future work can begin to fill the gaps. The goal was to provide a sufficient evidence base to facilitate rapid decision making by HTA bodies and regulators. Given the potentially significant public health impact of a disease-modifying treatment for AD, many HTA bodies and regulators have a keen interest in this field.

\subsection{Priority Outcomes}

An 'outcome' can be defined as the result of an intervention [5] and for a drug the most important outcome is the extent to which it maintains or improves health and well-being. The EXAG agreed that regulators will usually approve drugs based on a demonstrated effect on a certain clinical endpoint (preferably measured within an RCT), whereas HTA bodies will also consider and tend to prefer long-term outcomes that will include health-related quality of life, life expectancy, healthcare costs and (depending on the HTA body) societal

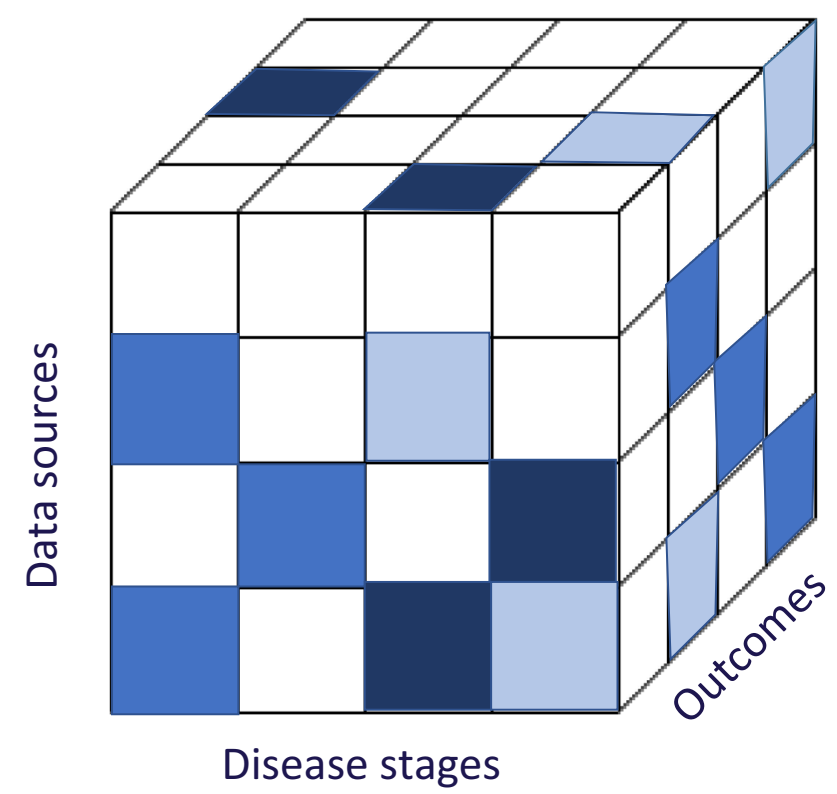

Fig. 1 The ROADMAP Data Cube visualises the project's 3 key activities and how they contributed to identify key gaps across Alzheimer's disease stages, outcomes, and data sources costs. For a new disease-modifying therapy in $\mathrm{AD}$, these are the outcomes that can be expected to be considered relevant.

Late-stage AD is characterised by cognitive impairment, functional decline and behavioural disturbances [6] and with further progression of the disease, institutionalisation/ full-time care also becomes an important outcome. In the National Institute for Health and Care Excellence appraisal of 'Donepezil, galantamine, rivastigmine and memantine for the treatment of Alzheimer's disease' [7], outcomes of interest included measures of disease severity, response to treatment, behavioural symptoms, mortality, the ability to remain independent, admission to full-time care, the healthrelated quality of life of patients and carers, and adverse effects of treatment. The four drugs covered by the appraisal were approved either by national regulatory agencies (donepezil, galantamine) or the EMA (rivastigmine, memantine). The primary outcomes cited in the regulatory assessments related to cognition, which is usually measured by the Alzheimer's Disease Assessment Scale-Cognition Subscale, and functioning; quality of life and other drivers for HTA were not included. Cognition was not considered as a primary outcome for memantine because in people with moderate-tosevere $\mathrm{AD}$, cognition would be difficult to assess and minor changes would be inconsequential unless they translated into global and functional improvements [8].

For the earlier disease stages of pre-clinical and prodromal $\mathrm{AD}$, outcomes related to cognition and the rate of cognitive decline may be more important than functioning, as functioning might not yet be severely affected in the earliest disease stages. This has implications for the regulatory and HTA context, as the primary outcomes of RCTs as well as their patient populations might well differ from those used for the approval of currently marketed drugs. For example, the phase III trials for solanezumab (a disease-modifying drug that failed to demonstrate efficacy) all had cognitive decline as measured by the Alzheimer's Disease Assessment Scale-Cognition Subscale as a primary endpoint [9]. As a result of the variety and complexity of cognitive-related outcomes, the EXAG discussed that a new consensus may be required that will lead to the development of new pharmacoeconomic models that are able to model the entire disease spectrum, including the prodromal and early AD stages.

The EXAG discussions (Table 1) identified that valid and well-established outcome measures in the early, often asymptomatic, disease stages are lacking, making defining and measuring the most relevant outcomes in the pre-clinical and prodromal disease stages a key priority for ROADMAP. A review of priority outcomes for key stakeholder groups performed by ROADMAP has been published [10]. The effectiveness of a disease-modifying drug in the pre-clinical disease stage will be driven by the ability to either prevent or delay clinical symptoms, resulting in a higher prominence for cognitive symptoms rather than functional impairment, 
Table 1 ROADMAP (real-world outcomes across the Alzheimer's disease spectrum: a multi-modal data access platform) expert advisory group recommendations

\begin{tabular}{|c|c|}
\hline Topic & Recommendations \\
\hline Priority outcomes & $\begin{array}{l}\text { Establish the rationale and justification for the selection of priority outcomes in pre-clinical AD and prodromal AD/MCI } \\
\text { Establish accepted outcomes for regulators, HTAs and payers for (1) defining prevention of AD and (2) delayed AD } \\
\text { onset } \\
\text { Ensure that instruments used for measuring these outcomes can distinguish between AD and other forms of dementia } \\
\text { Explore how modern technology (apps, wearables) could assist in measuring cognition that could easily be used across } \\
\text { settings } \\
\text { Establish the caregiver-relevant outcomes (quality of life, health status, loss of income, carer time) that are important to } \\
\text { capture for economic modelling }\end{array}$ \\
\hline $\begin{array}{l}\text { Disease progres- } \\
\text { sion and economic } \\
\text { modelling }\end{array}$ & $\begin{array}{l}\text { Real-world evidence will be required to support modelling assumptions as not all evidence to support modelling } \\
\text { assumptions (including caregiver impacts, use of endpoints, correlation between disease stages and certain outcomes) } \\
\text { can exclusively be generated from RCTs } \\
\text { Sensitivity analysis will be necessary to examine the robustness and impact of modelling assumptions on outcomes } \\
\text { The data cube approach will allow ROADMAP to identify current gaps in available data from different data sources and } \\
\text { will identify activities needed to fill those gaps } \\
\text { Better evidence is needed on the impact on caregivers in the different stages of the disease, both in the earlier stages } \\
\text { when the impact might be minimal, and in the later stages where the quality of life of caregivers might be substantially } \\
\text { affected, and how this impact can be valued } \\
\text { The degree of uncertainty arising from a switch from patient-reported to proxy-reported quality-of-life outcomes, par- } \\
\text { ticularly as these perspectives are not always compatible, will need to be examined } \\
\text { A pharmacoeconomic model will have to be able to accommodate for differences between national and regional settings } \\
\text { (i.e. delivery of care, relevant costs and outcomes to include) } \\
\text { Real-world data will be required to provide the required regional and national information }\end{array}$ \\
\hline
\end{tabular}

$A D$ Alzheimer's disease, $M C I$ mild cognitive impairment, $R C T$ s randomised controlled trials

which normally only becomes more apparent during the later stages of the disease. The EXAG discussed what a meaningful delay in disease progression might look like, but agreed that once a drug went through regulatory and HTA processes, this would depend on whether the drug's effect would be considered clinically relevant and what evidence would be available to support this. Therefore, the EXAG found that further work is needed to establish the best instruments to reliably measure cognition in people with $\mathrm{AD}$ in the pre-clinical stages. Furthermore, it will be essential to ensure that these instruments will not just be applicable in RCTs but, ideally, cognition should be measured using instruments that can measure cognition across settings including in the home environment.

Existing regulatory procedures can help validate and build a consensus on new outcome measures in AD. The EMA offers a procedure for the qualification of novel methods that can assess new methods for use in drug development, and joint qualification advice with HTA bodies is offered. The EXAG stressed the importance of pursuing such strategies once ROADMAP's outputs had matured, as the EXAG discussions are not intended to replace official procedures or advice. Therefore, existing routes for the validation of endpoints, instruments and disease models will need to be followed.

\subsection{Disease Progression and Economic Modelling}

With the identification of changes in the brain that can be identified many years before the first AD symptoms develop, the disease staging of AD has shifted and is now understood to have a long preclinical phase during which people show no signs of the disease but can test positive for disease markers. This can be used to identify people at risk of developing $\mathrm{AD}$ and therefore might be candidates for treatment with disease-modifying therapies. Alzheimer's disease stages across the disease continuum and AD outcomes are closely related, as within models disease stages are usually defined by outcomes, although the people in the preclinical disease stage are defined by testing positive for amyloid, tau or both.

Disease stages in previous disease progression models have been defined by the Mini-Mental State Examination score or by institutionalisation. For many diseases, economic modelling results in an increase in the amount of uncertainty surrounding outcomes over the model's time horizon. In $\mathrm{AD}$, the situation is the reverse: there is high uncertainty (owing to a lack of data) about the disease and how it affects outcomes in the pre-clinical and earlier disease stages - the starting point of a full disease progression model, whereas the later stages of the disease are relatively better characterised. This has a large impact on extrapolations of long-term 
effects based on short-term outcomes and therefore having data available that can mitigate some of this uncertainty is critical. The EXAG found that there is a role for real-world evidence to mitigate some of this uncertainty, but the data cube identified gaps between the outcomes relevant across the disease stages and those commonly measured in clinical practice.

\subsection{Regulatory vs. Health Technology Assessment Considerations}

The EXAG has members with regulatory as well as with HTA expertise, which proved helpful in discussing divergent requirements. The EXAG discussed the use of different endpoints for regulatory and HTA purposes, especially the preference for health-related quality of life from the HTA perspective. Historically, this was not a prominent endpoint in regulatory assessments that tended to focus more on clinical endpoints. Yet, it was noted that from a regulatory point of view, health-related quality of life could be considered in regulatory assessments if manufacturers would choose to make it a more prominent endpoint in their studies. The EXAG stipulated that impacts on cognition and functioning will be important from a regulatory perspective, and that HTA bodies will be interested in the clinical significance of such impacts and the cost effectiveness of the treatment.

In their assessments, several HTA bodies will not merely consider the impact of AD on people with the disease but will also include the impact the disease has on a caregiver's quality of life. Regulators will assess whether they consider the benefit-risk profile of a product positive or not. The EXAG noted that in theory, regulators could consider the impact on caregivers if there is compelling evidence that this should be considered as part of the benefit-risk profile of the product. These discussions illustrate the benefit of having a multi-disciplinary advisory group where questions are considered by regulatory and HTA experts simultaneously.

\subsection{Differences in National and Regional Healthcare Settings}

Once AD has progressed to a more severe disease stage, people generally will need various types of care, including unpaid and long-term formal care provided at home or in an institutional setting. The delivery and availability of different types of care will vary considerably between countries. However, within countries, there can also be substantial differences in the availability of care and the impact of the disease on patients and carers. The EXAG found that a health economic model would need to be adapted according to the setting used to evaluate a disease-modifying drug, given the differences in preferences between HTA bodies.

\subsection{Real-World Evidence}

ROADMAP has mapped different possible sources of realworld evidence across disease stages and identified a number of gaps that were discussed with the EXAG. Gaps that were identified included the use of different measures for cognition and functioning in RCTs vs. instruments that are used in clinical practice, the limited collection of biomarker status in clinical practice and a lack of follow-up of disease progression on disease instruments (such as the Mini-Mental State Examination) once an initial diagnosis is made. The EXAG noted that there is a lack of standardisation in the collection of real-world data and that there is a need to establish a consensus on what the most important data are in the context of $\mathrm{AD}$, such that improvements in data collection efforts can be made and gaps can be filled. The EXAG noted that existing procedures, such as the qualification of novel methods and scientific advice (offered by the EMA and with the possibility to involve HTA bodies), would allow for the validation of outcome measures and a disease progression model in AD.

Real-world evidence can be used for many different purposes and is used in HTAs for establishing prevalence, longterm outcomes and costs [11], but is not often used for establishing the comparative effectiveness of new drugs, although HTA bodies do sometimes accept real-world evidence when RCT data are not available [12]. There are limitations to the use of real-world evidence for a drug's effectiveness, and most HTA bodies place real-world evidence below RCT data in terms of the quality of evidence, stipulating that it should be used to confirm or supplement, rather than substitute RCT data [12]. The EXAG highlighted these limitations in its discussions and in discussing the role of real-world evidence, but did note that there might be a role for real-world evidence in $\mathrm{AD}$, for example, in providing evidence on the impact of the disease on carers, resource use and disease progression.

\section{Conclusions}

During the ROADMAP project, five different meetings with its EXAG explored a number of regulatory and HTA considerations for a future disease-modifying drug, of which the most important are summarised in this article (Table 1). ROADMAP was part of a group of Innovative Medicines Initiative projects called the Big Data for Better Outcomes Programme and, therefore, the project also explored the role that real-world evidence can play in $\mathrm{AD}$, and specifically, in supporting new therapies. Notwithstanding, in light of all the challenges of bringing a disease-modifying drug for $\mathrm{AD}$ to patients, ROADMAP and its focus on the role of real-world evidence is only a part of a bigger puzzle, and other research projects, for example those that are aimed at improving our 
understanding of the role biomarkers play in disease progression, are needed.

Many of the challenges that the EXAG identified could be solved by generating better real-world data and by better utilising existing data sources in AD. Therapies that target the earlier phases of the disease will have inherent uncertainties about the long-term impacts on patients and caregivers, but also on healthcare systems. Therefore, there is a clear need to agree and establish an international consensus on the AD outcomes that will facilitate and inform regulatory and HTA decision making, which has been initiated within ROADMAP. The challenge will be to ensure that data sources across Europe are able to generate the evidence needed to support regulatory and HTA decision making. Filling the gaps in the ROADMAP data cube would provide a step change in preparing Europe's healthcare systems for the future disease-modifying agents that are so urgently needed.

Acknowledgements The authors thank all ROADMAP colleagues who have contributed to the discussions reported in this article.

\section{Compliance with Ethical Standards}

Funding The activities reported in this article were conducted as part of the ROADMAP project. This project has received funding for the Innovative Medicines Initiative 2 Joint Undertaking under Grant Agreement No. 116020. The views and opinions expressed in this article are those of the authors only and cannot be understood as being made on behalf of, or reflecting the position or view of any of the authors' employing organisations. This article is not intended to replace or complement official guidelines that may be in place or in development. The open access fee was paid for by the ROADMAP project.

Conflict of interest Jacoline C. Bouvy, Pall Jonsson, Diana O'Rourke, Niklas Hedberg, Amr Makady, Entela Xoxi, Christine Gispen-de Wied, Anja Schiel, Raj Long and John Gallacher have no conflicts of interest directly relevant to the contents of this article (other than those specified under Funding). Antonella Santuccione Chadha is an employee of Roche Diagnostics.

Open Access This article is distributed under the terms of the Creative Commons Attribution-NonCommercial 4.0 International License (http://creativecommons.org/licenses/by-nc/4.0/), which permits any noncommercial use, distribution, and reproduction in any medium, provided you give appropriate credit to the original author(s) and the source, provide a link to the Creative Commons license, and indicate if changes were made.

\section{References}

1. Cummings J, Fox N. Defining disease modifying therapy for Alzheimer's disease. J Prev Alzheimers Dis. 2017;4(2):109-15.

2. Richie CW, Russ TC, Banerjee S, et al. The Edinburgh Consensus: preparing for the advent of disease-modifying therapies for Alzheimer's disease. Alzheimer's Res Ther. 2017;9:85.

3. Mehta D, Jackson R, Paul G, Shi J, Sabbagh M. Why do trials for Alzheimer's disease drugs keep failing? A discontinued drug perspective for 2010-2015. Expert Opin Investig Drugs. 2017;26(6):735-9.

4. ROADMAP. Advisory group. Available from: https://roadmapalzheimer.org/expert-advisory-group/. Accessed 2 Jan 2018.

5. International Consortium for Health Outcomes Measurement. What are outcomes? Available from: http://www.ichom.org/frequ ently-asked-questions/. Accessed 2 Jan 2018.

6. Isik AT. Late onset Alzheimer's disease in older people. Clin Interv Aging. 2010;5:307-11.

7. National Institute for Health and Care Excellence. Donepezil, galantamine, rivastigmine and memantine for the treatment of Alzheimer's disease. Technology appraisal TA217. Published 23 March 2011; last updated 11 May 2016. https://www.nice.org.uk/ guidance/ta217. Accessed 2 Jan 2018.

8. European Medicines Agency. Memantine scientific discussion. London, 15 November 2005. EMEA/H/C/463/II/15. http://www. ema.europa.eu/docs/en_GB/document_library/EPAR_-_Scien tific_Discussion_-_Variation/human/000463/WC500058762.pdf. Accessed 2 Jan 2018.

9. Siemers ER, Sundell KL, Carlson C, et al. Phase 3 solanezumab trials: secondary outcomes in mild Alzheimer's disease patients. Alzheimers Dement. 2016;12:110-20.

10. Nelson M, Smith M, Ly A, et al. Combined report: D2.3 Stakeholder generated lists of priority RWE relevant outcomes and D2.4 Disease progression and outcomes classification matrix. 2018. https://roadmap-alzheimer.org/wp-content/uploads/2018/07/ ROADMAP_D2.3D2.4.pdf. Accessed 10 Jan 2018.

11. Makady A, van Veelen A, Jonsson P, et al. Using real-world data in health technology assessment (HTA) practice: a comparative study of five HTA agencies. Pharmacoeconomics. 2018;36(3):359-368.

12. Makady A, ten Ham R, de Boer A, Hillege H, Klungel O, Goettsch W. Policies for use of real-world data in health technology assessment (HTA): a comparative study of six HTA agencies. Value Health. 2017;20(4):520-32. 